\title{
Investigação das propriedades psicométricas do Duke Religious Index no âmbito da pesquisa em Saúde Coletiva
}

\author{
Assessment of the psychometric properties of the Duke Religious \\ Index scale in the context of the Public Health research
}

\author{
Edson Zangiacomi Martinez ${ }^{1}$, Adorama Candido Alves², \\ Ariadne Fernanda Tesarin Mendes Carneiro ${ }^{3}$, Tatiane Martins Jorge ${ }^{4}$, \\ Antonio Carlos Duarte de Carvalho ${ }^{5}$, Miriane Lucindo Zucoloto ${ }^{6}$
}

\begin{abstract}
Resumo
A religiosidade é uma variável importante para a pesquisa em saúde, considerando suas conhecidas associações com o processo saúde-doença e questões importantes sobre o bem-estar, atitudes, práticas e satisfação em relação a cuidados e serviços. No entanto, na pesquisa em Saúde Coletiva, é frequentemente usada a afiliação religiosa como única dimensão da religiosidade. O presente estudo objetivou investigar a adequação do uso do instrumento Duke Religious Index (DUREL) no âmbito da pesquisa em Saúde Coletiva, ao avaliar as capacidades psicométricas do instrumento quando aplicado a uma população representativa de usuários dos serviços de atenção primária à saúde de um município de porte médio do interior do Estado de São Paulo. Foi encontrada uma adequada consistência interna para o instrumento e validade convergente-discriminante. Os resultados sugerem que o uso do DUREL não é restrito à pesquisa em saúde mental e que sua utilização pode trazer novas perspectivas para o entendimento de eventos de interesse na área da Saúde Coletiva.

Palavras-chave: religião e medicina; questionários; estudos de validação.

Abstract

Religiosity is an important variable in health research, given its known association with the health-disease process and important questions about the well-being, attitudes, practices and satisfaction with care and services. However, in the research in Public Health, it is often used religious affiliation as the only dimension of religiosity. This study aims to investigate the appropriateness of using the Duke Religious Index scale (DUREL) in the context of the Public Health research, evaluating the psychometric capabilities of the instrument when applied to a representative sample of users of primary care services to health in a medium-sized city of the interior of São Paulo State, Southeast Brazil. It was found an adequate internal consistency for the instrument and satisfactory convergent-discriminant validity. The results suggest that the use of DUREL is not restricted to research in mental health, and that its use can bring new perspectives for the understanding of events of interest in the field of Public Health.
\end{abstract}

Keywords: religion and medicine; questionnaires; validation studies.

\footnotetext{
Trabalho interdepartamental desenvolvido na Faculdade de Medicina de Ribeirão Preto, Universidade de São Paulo (FMRP/USP) - Ribeirão Preto (SP), Brasil. 1Professor Associado do Departamento de Medicina Social, FMRP/USP - Ribeirão Preto (SP), Brasil. ${ }^{2}$ Mestranda do Programa de Pós-Graduação em Saúde na Comunidade, FMRP/USP - Ribeirão Preto (SP), Brasil.

${ }^{3}$ Aprimoranda em Fonoaudiologia do Departamento de Oftalmologia, Otorrinolaringologia e Cirurgia da Cabeça e Pescoço, FMRP/USP - Ribeirão Preto (SP), Brasil. 4Professora da Divisão de Fonoaudiologia do Departamento de Oftalmologia, Otorrinolaringologia e Cirurgia da Cabeça e Pescoço, FMRP/USP - Ribeirão Preto (SP), Brasil.

${ }^{5}$ Professor Doutor do Departamento de Medicina Social, FMRP/USP - Ribeirão Preto (SP), Brasil.

${ }^{6}$ Doutoranda do Programa de Pós-Graduação em Saúde na Comunidade, FMRP/USP - Ribeirão Preto (SP), Brasil.

Endereço para correspondência: Edson Zangiacomi Martinez - Departamento de Medicina Social, Faculdade de Medicina de Ribeirão Preto - Universidade de São

Paulo - Avenida Bandeirantes, 3.900 - Monte Alegre - CEP: 14049-900 - Ribeirão Preto (SP), Brasil - E-mail: edson@fmrp.usp.br

Fonte de financiamento: nenhuma.

Conflito de interesses: nada a declarar.
} 


\section{INTRODUÇÃO}

A pesquisa em Saúde Coletiva objetiva a produção de conhecimentos relacionados à saúde da população que sejam capazes de auxiliar a elaboração de alternativas para a prevenção das doenças, a promoção da saúde e a organização de um sistema equânime de saúde ${ }^{1}$. No sentido de organizar os saberes produzidos e os conteúdos destinados ao ensino, podemos dispor a pesquisa em Saúde Coletiva em três diferentes campos: a epidemiologia, as ciências sociais em saúde e as políticas, o planejamento e a gestão da saúde ${ }^{2}$. Nesses três contextos, os estudos de natureza empírica geralmente buscam relacionar as condições e os problemas de saúde das pessoas e grupos humanos com variáveis biológicas, como sexo, idade, peso corporal, número de gestações e etnia, ou socioculturais, como estado civil, procedência geográfica, classe social e escolaridade. Entre essas variáveis socioculturais é possível incluir a religião ${ }^{3} \mathrm{e}$ a religiosidade. Na conceituação do sociólogo Émile Durkheim (1858-1917), a religião pode ser entendida como "um sistema de crenças e práticas em relação ao sagrado, que unem em uma mesma comunidade moral todos os que a ela aderem"4. Este conceito não é distante daquele apresentado por Koenig et al., em que "religião é o sistema organizado de crenças, práticas, rituais e símbolos designados para facilitar o acesso ao sagrado (Deus, força maior, verdade suprema)" ${ }^{3,6}$. Esses consequentes ritos, símbolos e valores contribuem, em parte, para as percepções do indivíduo quanto ao ambiente e a sociedade, o que pode determinar seus comportamentos e suas atitudes. No âmbito da saúde, a religião oportuniza então efeitos coletivos, como mostra o estudo de Olinto e Moreira-Filho ${ }^{7}$, apontando que mulheres que não professam nenhuma religião tem um risco maior de praticar aborto, quando comparadas com as mulheres de religião católica. Outros estudos destacam as relações entre a religião e as percepções e atitudes sobre outras questões relevantes em saúde, como eutanásia ${ }^{8,9}$, doação de órgãos ${ }^{10,11}$, tabagismo ${ }^{12}$ e etilismo ${ }^{13,14}$. A religião pode exercer efeito também sobre a fecundidade pré-marital na adolescência ${ }^{15}$, a satisfação de pacientes em relação a cuidados recebidos em saúde ${ }^{16} \mathrm{e}$ taxas de mortalidade ${ }^{17}$.

A religiosidade, por sua vez, é definida por Koenig et al. como "o quanto um indivíduo acredita, segue e pratica uma determinada religião"5,6. Dentre as muitas implicações da religiosidade no processo saúde-doença, Linard et al. ${ }^{18}$ destacam que ela serviu como suporte para um grupo de mulheres portadoras de câncer de colo uterino enfrentar o desafio de viver com a incerteza da cura. A religiosidade também é um modulador importante no consumo de álcool e drogas entre estudantes adolescentes, segundo Dalgalarrondo et al. ${ }^{19}$.
Além disso, segundo Sanchez e Nappo ${ }^{20}$, pessoas que regularmente frequentam um culto religioso ou que praticam no seu cotidiano as propostas da religião professada tendem a apresentar menores índices de consumo de drogas lícitas e ilícitas e a apresentar melhor recuperação quando seu tratamento é acompanhado por uma abordagem espiritual, de qualquer origem. A religiosidade, em sua definição, diferese da espiritualidade, outra dimensão individual bastante utilizada em estudos atuais em saúde. Na conceituação de Koenig et al., a espiritualidade

se refere a uma busca pessoal de compreensão relacionada a questões existenciais maiores, como o fim e o sentido da vida, e suas relações com o sagrado e/ou transcendente ${ }^{5}$.

São evidentes as possíveis intersecções entre os conceitos de religiosidade e de espiritualidade, mas esta última não necessariamente leva o indivíduo a desenvolver práticas religiosas ou mesmo formar comunidades religiosas, de acordo com Taunay et al. ${ }^{6}$. Ou ainda, segundo Panzini et al. ${ }^{21}$, a religiosidade sugere um sistema de adoração ou uma doutrina específica participada em um grupo.

Para uma conceituação mais objetiva da religiosidade, Koenig et al. ${ }^{22,23}$ descrevem três principais dimensões do envolvimento religioso em relação às suas implicações com desfechos de interesse em saúde. Definem-se então as religiosidades organizacional (RO), privada ou não organizacional (RNO) e intrínseca (RI). A RO considera a frequência a encontros religiosos, como cultos, missas, cerimônias e grupos de oração. Está, portanto, relacionada a um componente social. A RNO engloba os hábitos e comportamentos religiosos que podem ocorrer fora do ambiente e do contexto de uma instituição ou organização religiosa, sem a forma das liturgias estabelecidas por estas. Ao contrário da RO, não depende necessariamente da interação com outras pessoas, podendo manifestar-se individualmente ou em pequenos grupos familiares e informais ${ }^{24}$. Encontra exemplos nas orações, na leitura da Bíblia ou de outros textos religiosos e na audiência a programas religiosos da televisão ou do rádio. Na RI a religião assume um significado em que todos os aspectos da vida são compreendidos em função dela. Refere-se à "busca de internalização e vivência plena da religiosidade como principal objetivo do indivíduo"'. Enquanto na religiosidade extrínseca a religião é "um meio utilizado para obter outros fins ou interesses, para proporcionar segurança e consolo, sociabilidade e distração, status e auto-absolvição", conforme Stroppa e Moreira-Almeida ${ }^{25}$, a RI tem por foco a crença religiosa em si, sem interesse primário em outros ganhos, autopromoção ou satisfação de convenções sociais. 
O Duke Religious Index (DUREL) ${ }^{22}$ é uma breve escala que capta essas três dimensões da religiosidade. O instrumento possui cinco itens, sendo os dois primeiros relativos a RO e RNO. Os três itens restantes foram adaptados de um instrumento anteriormente introduzido por $\mathrm{Hoge}^{26}$, relativo à RI. Alguns subsequentes estudos mostraram que o instrumento possui satisfatórias propriedades psicométricas quando aplicado a pacientes oncológicos $^{27}$ e estudantes universitários ${ }^{28}$. O DUREL foi traduzido em língua portuguesa por Moreira-Almeida et al. ${ }^{29}$, sendo essa versão chamada P-DUREL, e suas propriedades psicométricas foram então estudadas por Taunay et al. ${ }^{6}$, Lucchetti et al. ${ }^{30} \mathrm{e}$ Martinez et al. ${ }^{31} \mathrm{Na}$ versão em português, o primeiro item do instrumento pergunta: "Com que frequência você vai a uma igreja, templo ou outro encontro religioso?", sendo as possíveis respostas oferecidas em uma escala de seis pontos, de "Mais do que uma vez por semana" a "Nunca". O segundo item é "Com que frequência você dedica o seu tempo a atividades religiosas individuais, como preces, rezas, meditações, leitura da bíblia ou de outros textos religiosos?", cujas possíveis respostas também compõem uma escala de seis pontos, de "Mais do que uma vez ao dia" a "Raramente ou nunca". Os três itens restantes levam as seguintes frases aos respondentes: "Em minha vida, eu sinto a presença de Deus (ou do Espírito Santo)", "As minhas crenças religiosas estão realmente por trás de toda a minha maneira de viver" e "Eu me esforço muito para viver a minha religião em todos os aspectos da vida". Os respondentes são então convidados a assinalar o quanto essas frases se aplicam a eles, em uma escala de cinco pontos, de "Totalmente verdade para mim" a "Não é verdade". A mensuração da RI é então a soma das pontuações obtidas nesses três itens, sendo o número máximo de pontos igual a 15 .

O DUREL tem sido bastante utilizado na pesquisa em psiquiatria e saúde mental ${ }^{32-35}$, mas seu uso na pesquisa em Saúde Coletiva ainda é discreto. Portanto, o presente estudo objetivou investigar a adequação do uso do P-DUREL no âmbito da pesquisa em Saúde Coletiva, ao avaliar as capacidades psicométricas do instrumento quando aplicado a uma população representativa de usuários dos serviços de atenção primária à saúde de um município de porte médio do interior do Estado de São Paulo.

\section{METODOLOGIA}

O presente estudo transversal é parte de um projeto de pesquisa maior que pretende investigar conhecimentos, crenças e práticas da medicina popular em moradores adultos (usuários de serviços públicos de saúde) de Ribeirão Preto. Este projeto de pesquisa e seu respectivo Termo de Consentimento Livre e Esclarecido (TCLE) foram apreciados e aprovados pelo Comitê de Ética em Pesquisa do Hospital das Clínicas da Faculdade de Medicina de Ribeirão Preto da Universidade de São Paulo (parecer n 258.211, de 23 de abril de 2013).

\section{Plano amostral}

Este estudo teve como alvo a população adulta, moradora de Ribeirão Preto, Estado de São Paulo, usuária de unidades de saúde do município. A população do município no ano de 2010 era de 604.682 habitantes, segundo o Censo Demográfico do Instituto Brasileiro de Geografia e Estatística (IBGE, http://cidades.ibge.gov.br/). A área urbana do município é dividida em cinco distritos de saúde, Norte, Oeste, Central, Sul e Leste. Em cada um desses distritos é localizada uma Unidade Básica Distrital de Saúde (UBDS), que é a referência para algumas especialidades médicas naquela região. $\mathrm{Na}$ ocasião da coleta dos dados, havia no município 40 unidades de saúde, classificadas em UBDSs, Unidades de Saúde da Família (USFs) e Unidades Básicas de Saúde (UBSs). Um mapa do município localizando as regiões de abrangência dessas unidades de saúde é apresentado por Roza et al. ${ }^{36}$. As unidades de saúde foram então agrupadas em 10 estratos, de acordo com o distrito em que se localizam e o Índice Paulista de Vulnerabilidade Social (IPVS) predominante, sendo agrupadas em um mesmo estrato áreas com IPVS predominante igual a 1 ou 2 , e em um mesmo estrato IPVS predominante igual ou maior do que 4. O IPVS, com rótulos de 1 a 6 , classifica uma área como, respectivamente, de nenhuma vulnerabilidade, vulnerabilidade muito baixa, vulnerabilidade baixa, vulnerabilidade média, vulnerabilidade alta ou vulnerabilidade muito alta. Por fim, foi sorteada uma unidade de saúde de cada estrato, onde as entrevistas foram conduzidas. Este plano de amostragem objetivou, portanto, uma amostra que representasse a população usuária de unidades de saúde do município sem deixar de considerar a heterogeneidade que existe em termos de vulnerabilidade social. O tamanho amostral foi obtido considerando um plano amostral estratificado ${ }^{37}$, um intervalo de credibilidade de 95\% (IC95\%) e uma precisão absoluta de $4 \%$ para a estimativa da proporção de pessoas que possuem alguma crença na medicina popular, cuja estimação é o objetivo principal do projeto maior em que o presente estudo se insere. Tal proporção, para maximização da variância, foi considerada igual a 50\% em cada estrato. Determinou-se, dessa maneira, um tamanho amostral mínimo de 605 entrevistas, sendo o número de entrevistas a ser realizado em cada estrato proporcional ao seu respectivo tamanho populacional.

\section{Coleta dos dados}

As entrevistas individuais foram conduzidas nas salas de espera das unidades de saúde, por duas entrevistadoras treinadas, 
com o aval do respectivo coordenador ou gestor. Após uma breve apresentação dos propósitos da pesquisa, as entrevistadoras solicitavam a assinatura do TCLE e iniciavam o roteiro de aplicação dos instrumentos.

\section{Análise estatística}

Modelos de regressão múltipla baseados na distribuição beta-binomial $^{38}$ foram utilizados para estudar a associação entre os escores do P-DUREL e as seguintes variáveis: sexo, faixa etária, escolaridade, filiação religiosa declarada e autopercepção do estado de saúde (classificada em boa, regular e ruim). Esses modelos foram utilizados considerando que os escores do P-DUREL são representados por variáveis quantitativas discretas que assumem um número finito de possibilidades, o que torna a distribuição beta-binomial adequada aos dados da escala ${ }^{39}$. Estimativas dos coeficientes dos modelos e seus respectivos valores de IC95\% foram obtidos por métodos de inferência bayesiana ${ }^{40}$ com distribuições, a priori, não informativas e com o auxílio de simulação Monte Carlo em Cadeia de Markov (MCMC). Os intervalos de credibilidade bayesianos são análogos aos intervalos de confiança obtidos pelo método frequentista de estatística. Quando um IC95\% para um coeficiente do modelo contém o valor zero, infere-se que a respectiva variável não se associa significativamente com os escores do P-DUREL ( similar a $\mathrm{p}<0,05)$.

Para cada dimensão do P-DUREL, foram calculadas as médias e os desvios-padrão amostrais, o efeito chão e o efeito teto ("floor and ceiling effects"). Os efeitos chão e teto são definidos como a porcentagem de sujeitos que tiveram o escore mais baixo ou mais alto em cada dimensão, respectivamente. O número máximo de pontos para cada dimensão é denotado por k, de forma que ké igual a 6 para as dimensões RO e RNO e igual a 15 para a dimensão RI. Dessa forma, considera-se a situação de máxima uniformidade das respostas à dimensão quando as frequências relativas a cada possível escore é $1 / \mathrm{k}$. Um efeito teto ou chão é então considerado substancial quando superior a $1 / \mathrm{k}$ (ou seja, superior a $17 \%$ para as dimensões $\mathrm{RO}$ e RNO, ou superior a 7\% para a RI).

Para análise de consistência interna do P-DUREL aplicado à população em estudo, foi determinado o valor do coeficiente alfa de Cronbach ${ }^{41}$. Para a busca de evidência de validade convergente-discriminante, foram calculados coeficientes de correlação de Spearman entre as três subescalas do P-DUREL ${ }^{6}$. Uma análise fatorial exploratória (por componentes principais) foi utilizada para testar a hipótese de uma estrutura composta por um único fator ${ }^{28}$. O índice KaiserMeyer-Olkin (KMO) foi utilizado para descrever a adequação amostral nessa análise.

\section{RESULTADOS}

Foram entrevistados 605 indivíduos, sendo 414 mulheres, com idade entre 18 e 87 anos (média 38,4; desvio-padrão 15,4 anos), e 191 homens, com idade entre 18 e 85 anos (média 44,9; desvio-padrão 16,9 anos). A frequência maior de mulheres encontrada na presente amostra reflete um perfil já conhecido pela literatura ${ }^{42}$ : mulheres utilizam mais os serviços de saúde. A Tabela 1 apresenta uma descrição da amostra segundo o sexo, faixa etária, escolaridade, afiliação religiosa declarada e autopercepção do estado de saúde, classificada como ruim, regular ou boa. Nota-se uma frequência relativamente baixa de indivíduos analfabetos (2\%) e com ensino superior completo (9\%), sendo a maioria católica (58\%) e com estado de saúde autorreferido como "bom" (72\%). As frequências relativas observadas para cada uma das diferentes afiliações religiosas são próximas àquelas encontradas no Censo Demográfico de 2010 para a população de Ribeirão Preto (IBGE, disponível em http://cidades.ibge.gov.br/), em que o percentual de católicos é 60,8\%, de evangélicos, 19,9\%, de espíritas, 6,7\%, de ateus, 0,6\%, de Testemunhas de Jeová, $1,4 \%$, e de pessoas não vinculadas a alguma religião, 6,9\%. A Tabela 1 também exibe médias e desvios-padrão amostrais para as três dimensões do P-DUREL.

A Tabela 2 apresenta os resultados do ajuste dos modelos de regressão múltipla baseados na distribuição beta-binomial. Para esses modelos, uma estimativa menor do que zero para o coeficiente associado a uma classe de uma variável evidencia um escore médio menor do que aquele da respectiva classe de referência, enquanto coeficientes com estimativas positivas evidenciam médias maiores. Assim, observa-se que indivíduos de sexo feminino apresentam maiores escores médios para todas as três dimensões de religiosidade do P-DUREL. Observou-se um efeito expressivo da idade sobre as dimensões RNO e RI, de modo que indivíduos com 51 anos ou mais possuem maior RNO e indivíduos com mais de 60 anos possuem maior RI. Não foram observadas evidências de efeitos da escolaridade sobre as dimensões RO e RNO, mas é evidenciado que indivíduos com ensino médio completo possuem menor escore médio de RI. Quando comparados com os indivíduos católicos, evangélicos, espíritas e Testemunhas de Jeová apresentam maiores escores médios para todos os domínios do P-DUREL, enquanto os ateus e aqueles que acreditam em Deus sem possuir uma afiliação religiosa apresentam menores escores médios. A autopercepção do estado de saúde associou-se apenas com os escores de RO, de forma que os indivíduos que classificam sua saúde como ruim apresentam uma maior média para esta dimensão da religiosidade.

A Tabela 3 descreve os efeitos teto e chão para as três dimensões do instrumento, segundo as afiliações religiosas. 
Tabela 1. Caracterização da amostra e estatísticas descritivas (médias e desvios-padrão) para as dimensões da religiosidade mensuradas pelo Duke Religious Index (versão em português)

\begin{tabular}{|c|c|c|c|c|}
\hline \multirow[b]{2}{*}{ Variáveis } & \multirow[b]{2}{*}{ n (\%) } & \multicolumn{3}{|c|}{ Dimensões da religiosidade (P-DUREL) } \\
\hline & & $\begin{array}{c}\text { RO } \\
\text { Média (DP) }\end{array}$ & $\begin{array}{c}\text { RNO } \\
\text { Média (DP) }\end{array}$ & $\begin{array}{c}\text { RI } \\
\text { Média (DP) }\end{array}$ \\
\hline \multicolumn{5}{|l|}{ Sexo } \\
\hline Feminino & $414(68)$ & $4,14(1,46)$ & $4,69(1,22)$ & $12,83(2,79)$ \\
\hline Masculino & $191(32)$ & $3,46(1,63)$ & $4,04(1,65)$ & $11,79(3,42)$ \\
\hline \multicolumn{5}{|l|}{ Faixa etária } \\
\hline Até 25 anos & $119(20)$ & $3,85(1,57)$ & $4,35(1,43)$ & $12,26(3,09)$ \\
\hline 26 a 30 anos & $81(13)$ & $3,95(1,57)$ & $4,40(1,43)$ & $11,86(3,23)$ \\
\hline 31 a 40 anos & $133(22)$ & $3,92(1,46)$ & $4,50(1,37)$ & $12,33(3,03)$ \\
\hline 41 a 50 anos & $103(17)$ & $3,93(1,52)$ & $4,32(1,59)$ & $12,29(3,27)$ \\
\hline 51 a 60 anos & $75(12)$ & $4,03(1,67)$ & $4,73(1,25)$ & $13,08(2,61)$ \\
\hline Mais de 60 anos & $94(16)$ & $3,90(1,57)$ & $4,69(1,28)$ & $13,35(2,69)$ \\
\hline \multicolumn{5}{|l|}{ Escolaridade } \\
\hline Analfabeto & $14(2)$ & $4,50(1,34)$ & $4,50(1,65)$ & $14,07(1,73)$ \\
\hline Fundamental incompleto & $118(20)$ & $4,06(1,54)$ & $4,52(1,48)$ & $13,18(2,55)$ \\
\hline Fundamental completo & $164(27)$ & $3,94(1,51)$ & $4,45(1,41)$ & $12,44(3,16)$ \\
\hline Médio completo & $252(42)$ & $3,84(1,56)$ & $4,51(1,35)$ & $12,25(2,94)$ \\
\hline Superior completo & $57(9)$ & $3,82(1,60)$ & $4,40(1,45)$ & $12,00(3,93)$ \\
\hline \multicolumn{5}{|l|}{ Afiliação religiosa } \\
\hline Católica & $351(58)$ & $3,69(1,37)$ & $4,47(1,32)$ & $12,42(2,84)$ \\
\hline Evangélica & $150(25)$ & $4,89(1,18)$ & $4,94(1,03)$ & $13,61(2,11)$ \\
\hline Espírita & $41(7)$ & $4,42(1,13)$ & $4,88(1,02)$ & $13,85(1,75)$ \\
\hline Acredita em Deus & $39(6)$ & $1,92(1,48)$ & $3,08(1,84)$ & $8,87(3,00)$ \\
\hline Ateu & $10(2)$ & $1,00(0,00)$ & $1,00(0,00)$ & $3,22(0,67)$ \\
\hline Testemunha de Jeová & $12(2)$ & $5,58(0,51)$ & $5,25(0,87)$ & $14,83(0,39)$ \\
\hline Candomblé e Umbanda & $2(<1)$ & $4,50(0,71)$ & $5,00(0,00)$ & $14,50(0,71)$ \\
\hline \multicolumn{5}{|c|}{ Autopercepção do estado de saúde } \\
\hline Ruim & $18(3)$ & $3,06(1,59)$ & $4,22(1,77)$ & $11,56(3,76)$ \\
\hline Regular & $149(25)$ & $4,13(1,45)$ & $4,70(1,30)$ & $12,64(2,93)$ \\
\hline Boa & $438(72)$ & $3,89(1,56)$ & $4,42(1,42)$ & $12,49(3,05)$ \\
\hline
\end{tabular}

P-DUREL: Duke Religious Index (versão em português); RO: religiosidade organizacional; RNO: religiosidade não organizacional; RI: religiosidade intrínseca; DP: desvio padrão.

Não são observados efeitos teto e chão substanciais para as dimensões RO e RNO quando considerada toda a amostra, mas a dimensão RI apresenta um substancial efeito teto (41,2\%). Evangélicos e Testemunhas de Jeová apresentam substanciais efeitos teto nas três dimensões do P-DUREL, enquanto ateus apresentam um substancial efeito chão para as três dimensões. Efeitos chão substanciais são também observados para os indivíduos que acreditam em Deus sem pertencer a alguma afiliação religiosa, quando consideradas as dimensões RO e RNO.

Foi encontrado um coeficiente alfa de Cronbach de 0,822 para o P-DUREL e de 0,742 para a escala de RI, na amostra de 605 indivíduos. Foram encontrados coeficientes de correlação de Spearman de 0,49 entre as subescalas de RO e RNO, de 0,56 entre as subescalas RI e RO e de 0,41 entre RI e RNO $(\mathrm{p}<0,01)$. Em adição, um único fator com autovalor 2,9 foi retido em uma análise fatorial exploratória, explicando 58,6\% de variância. Um índice KMO de 0,78 indicou boa adequação amostral nessa análise.

A Tabela 4 sintetiza os resultados do presente estudo, comparando-os aos encontrados por Taunay et al. ${ }^{6}$, Lucchetti et al. ${ }^{30}$ e Martinez et al. ${ }^{31}$. Observa-se que, assim como nesses estudos de validação disponíveis na literatura, o presente estudo também exibe satisfatória capacidade psicométrica para o P-DUREL, quando aplicado a uma população de usuários de serviços de atenção primária. 
Tabela 2. Resultados do ajuste dos modelos de regressão beta-binomial, comparando as médias dos escores das dimensões da religiosidade mensuradas pelo Duke Religious Index (versão em português)

\begin{tabular}{|c|c|c|c|}
\hline & $\begin{array}{c}\text { RO } \\
\text { Coeficiente (IC95\%) }\end{array}$ & $\begin{array}{c}\text { RNO } \\
\text { Coeficiente (IC95\%) }\end{array}$ & $\begin{array}{c}\text { RI } \\
\text { Coeficiente (IC95\%) }\end{array}$ \\
\hline Intercepto & $0,550(-0,174-1,335)$ & $0,899(0,150-1,688)$ & $2,445(1,356-3,754)$ \\
\hline \multicolumn{4}{|l|}{ Sexo } \\
\hline Feminino & Ref. & Ref. & Ref. \\
\hline Masculino & $-0,397(-0,576--0,217)^{*}$ & $-0,472(-0,658--0,278)^{\star}$ & $-0,419(-0,642--0,202)^{*}$ \\
\hline \multicolumn{4}{|l|}{ Faixa etária } \\
\hline Até 25 anos & Ref. & Ref. & Ref. \\
\hline 26 a 30 anos & $-0,010(-0,298-0,285)$ & $-0,048(-0,362-0,268)$ & $-0,276(-0,623-0,077)$ \\
\hline 31 a 40 anos & $0,032(-0,227-0,302)$ & $0,153(-0,122-0,429)$ & $0,056(-0,264-0,368)$ \\
\hline 41 a 50 anos & $0,020(-0,257-0,309)$ & $0,038(-0,259-0,334)$ & $0,049(-0,314-0,389)$ \\
\hline 51 a 60 anos & $0,109(-0,204-0,436)$ & $0,375(0,035-0,711)^{\star}$ & $0,399(-0,027-0,829)$ \\
\hline Mais de 60 anos & $0,098(-0,204-0,407)$ & $0,464(0,135-0,795)^{*}$ & $0,717(0,284-1,152)^{*}$ \\
\hline \multicolumn{4}{|l|}{ Escolaridade } \\
\hline Analfabeto & Ref. & Ref. & Ref. \\
\hline Fundamental incompleto & $-0,388(-1,043-0,200)$ & $0,046(-0,605-0,660)$ & $-0,878(-2,017-0,077)$ \\
\hline Fundamental completo & $-0,396(-1,045-0,183)$ & $0,109(-0,552-0,717)$ & $-1,060(-2,206--0,123)$ \\
\hline Médio completo & $-0,446(-1,081-0,137)$ & $0,283(-0,383-0,897)$ & $-1,127(-2,269--0,177)^{\star}$ \\
\hline Superior completo & $-0,349(-1,042-0,273)$ & $0,196(-0,503-0,843)$ & $-1,012(-2,200-0,004)$ \\
\hline \multicolumn{4}{|l|}{ Afiliação religiosa } \\
\hline Católica & Ref. & Ref. & Ref. \\
\hline Evangélica & $1,000(0,781-1,226)^{\star}$ & $0,445(0,223-0,674)^{*}$ & $0,688(0,419-0,965)^{*}$ \\
\hline Espírita & $0,546(0,200-0,903)^{\star}$ & $0,364(0,003-0,740)^{*}$ & $0,852(0,356-1,402)^{*}$ \\
\hline Acredita em Deus & $-1,180(-1,507--0,846)^{\star}$ & $-0,983(-1,301--0,657)^{\star}$ & $-1,116(-1,466--0,744)^{\star}$ \\
\hline Ateu & $-1,865(-2,622--1,166)^{*}$ & $-2,495(-3,274--1,782)^{*}$ & $-2,481(-3,187--1,810)^{\star}$ \\
\hline Testemunha de Jeová & $2,126(1,244-3,168)^{\star}$ & $0,936(0,186-1,805)^{*}$ & $2,397(1,052-4,239)^{\star}$ \\
\hline Candomblé e Umbanda & $0,579(-0,923-2,369)$ & $0,334(-1,229-2,277)$ & $1,687(-0,378-4,658)$ \\
\hline \multicolumn{4}{|c|}{ Autopercepção do estado de saúde } \\
\hline Ruim & Ref. & Ref. & Ref. \\
\hline Regular & $0,513(0,022-0,996)^{*}$ & $0,181(-0,363-0,719)$ & $0,163(-0,501-0,794)$ \\
\hline Boa & $0,409(-0,071-0,874)$ & $-0,042(-0,553-0,475)$ & $0,266(-0,369-0,852)$ \\
\hline
\end{tabular}

*Intervalos de credibilidade que não incluem o valor zero indicam evidências de associação; RO: religiosidade organizacional; RNO: religiosidade não organizacional; RI: religiosidade intrínseca; IC95\%: intervalo de credibilidade de 95\%; Ref.: classe de referência.

Tabela 3. Efeitos teto e chão para as dimensões do Duke Religious Index (versão em português)

\begin{tabular}{|c|c|c|c|c|c|c|}
\hline \multirow[b]{2}{*}{ Afiliação religiosa } & \multicolumn{2}{|c|}{ RO } & \multicolumn{2}{|c|}{ RNO } & \multicolumn{2}{|c|}{ RI } \\
\hline & $\begin{array}{c}\text { Efeito chão } \\
\text { (\%) }\end{array}$ & $\begin{array}{c}\text { Efeito teto } \\
\text { (\%) }\end{array}$ & $\begin{array}{c}\text { Efeito chão } \\
(\%)\end{array}$ & $\begin{array}{c}\text { Efeito teto } \\
\text { (\%) }\end{array}$ & $\begin{array}{c}\text { Efeito chão } \\
(\%)\end{array}$ & $\begin{array}{c}\text { Efeito teto } \\
(\%)\end{array}$ \\
\hline Católica & 9,7 & 5,7 & 6,8 & 12,2 & 1,1 & $37,9^{*}$ \\
\hline Evangélica & 1,3 & $35,3^{*}$ & 1,3 & $26,7^{*}$ & 0,7 & $52,0^{*}$ \\
\hline Espírita & 2,4 & 14,6 & 2,4 & $24,4^{*}$ & 0 & $58,5^{\star}$ \\
\hline Acredita em Deus & $64,1^{\star}$ & 2,6 & $35,9^{*}$ & 0 & 2,6 & 7,7 \\
\hline Ateu & $100^{*}$ & 0 & $100^{*}$ & 0 & $88,9^{*}$ & 0 \\
\hline Testemunha de Jeová & 0 & $58,3^{*}$ & 0 & $41,7^{\star}$ & 0 & $83,3^{*}$ \\
\hline Toda a amostra & 11,9 & 14,4 & 8,4 & 16,2 & 2,3 & $41,2^{\star}$ \\
\hline
\end{tabular}

*Efeitos considerados substanciais; RO: religiosidade organizacional; RNO: religiosidade não organizacional; RI: religiosidade intrínseca. 
Tabela 4. Comparações entre os resultados do presente estudo e outros estudos de validação da escala Duke Religious Index (versão em português)

\begin{tabular}{|c|c|c|c|c|}
\hline Referência & População & $\mathbf{n}$ & Caracterização da amostra & Resultados \\
\hline Taunay et al. ${ }^{6}$ & $\begin{array}{l}\text { Estudantes universitários da } \\
\text { área da Saúde, dos cursos de } \\
\text { graduação em Psicologia e } \\
\text { Medicina de uma universidade } \\
\text { pública do Ceará. }\end{array}$ & 323 & $\begin{array}{c}\text { Idade média de } 20,7 \text { anos, } 66 \% \\
\text { mulheres, } 60 \% \text { católicos, } 12 \% \\
\text { evangélicos, } 8 \% \text { ateus ou agnósticos, } \\
8 \% \text { sem religião. }\end{array}$ & $\begin{array}{l}\text { Alfa de Cronbach igual a 0,89. } \\
\text { Adequada confiabilidade teste- } \\
\text { resteste. Correlação entre as } \\
\text { subescalas RI e RO, r=0,71; RI e } \\
\text { RNO, r=0,64; RO e RNO, r=0,62. }\end{array}$ \\
\hline Taunay et al. ${ }^{6}$ & $\begin{array}{l}\text { Pacientes psiquiátricos } \\
\text { atendidos no ambulatório de } \\
\text { psiquiatria de um Hospital } \\
\text { Universitário do Ceará. }\end{array}$ & 102 & $\begin{array}{l}\text { Idade média de } 40,4 \text { anos, } 69 \% \\
\text { mulheres, } 59 \% \text { católicos, } 12 \% \\
\text { evangélicos, } 7 \% \text { espíritas, } 21 \% \text { sem } \\
\text { religião; } 38 \% \text { só tinham o ensino } \\
\text { fundamental. }\end{array}$ & $\begin{array}{c}\text { Alfa de Cronbach }=0,87 \text {. Adequada } \\
\text { confiabilidade teste-resteste. } \\
\text { Correlação entre as subescalas RI e } \\
\text { RO, r=0,71; RI e RNO, r=0,58; RO e } \\
\text { RNO, r=0,62. }\end{array}$ \\
\hline Lucchetti et al. ${ }^{30}$ & $\begin{array}{c}\text { Indivíduos adultos, de } \\
\text { baixa renda, residentes na } \\
\text { comunidade de Paraisópolis, } \\
\text { São Paulo. }\end{array}$ & 383 & $\begin{array}{c}\text { Idade média de } 41,7 \text { anos, } 74 \% \\
\text { mulheres, } 72,6 \% \text { católicos, } 13,3 \% \\
\text { evangélicos, } 8,9 \% \text { sem religião, } 0,5 \% \\
\text { espíritas. }\end{array}$ & $\begin{array}{l}\text { Alfa de Cronbach igual a } 0,733 \text { para o } \\
\text { P-DUREL e de } 0,758 \text { para a escala RI. } \\
\text { Correlação entre as subescalas RI e } \\
\text { RO, r=0,37; RI e RNO, r=0,36; RO } \\
\text { e RNO, } r=0,46 .\end{array}$ \\
\hline Martinez et al. ${ }^{31}$ & $\begin{array}{l}\text { Estudantes universitários de } \\
\text { diferentes cursos de pós- } \\
\text { graduação da área da Saúde } \\
\text { de uma universidade pública } \\
\text { do Estado de São Paulo. }\end{array}$ & 160 & Sem informações. & $\begin{array}{c}\text { Alfa de Cronbach de } 0,88 \\
\text { para o P-DUREL e de } 0,89 \text { para } \\
\text { a escala RI. Correlação entre as } \\
\text { subescalas RI e RO, r=0,58; RI e } \\
\text { RNO, r=0,62; RO e RNO, r=0,58. }\end{array}$ \\
\hline Presente estudo & $\begin{array}{c}\text { Usuários dos serviços de } \\
\text { atenção primária à saúde } \\
\text { de Ribeirão Preto, Estado } \\
\text { de São Paulo. }\end{array}$ & 605 & $\begin{array}{c}\text { Idade média de } 40,5 \text { anos, } 68 \% \\
\text { mulheres, } 58 \% \text { católicos, } 25 \% \\
\text { evangélicos, } 7 \% \text { espíritas, } 8 \% \\
\text { sem religião. }\end{array}$ & $\begin{array}{c}\text { Alfa de Cronbach igual a } 0,822 \text { para o } \\
\text { P-DUREL e de } 0,742 \text { para a escala RI. } \\
\text { Correlação entre as subescalas RI e } \\
\text { RO, r=0,56; RI e RNO, r=0,41; RO } \\
\text { e RNO, } r=0,49 .\end{array}$ \\
\hline
\end{tabular}

RO: religiosidade organizacional; RNO: religiosidade não organizacional; RI: religiosidade intrínseca.

\section{DISCUSSÃO}

Em uma revisão da literatura concluída em 2011, Lucchetti et $a{ }^{43}$ mostraram que estão disponíveis 20 escalas para a mensuração da espiritualidade e/ou religiosidade, sendo três desenvolvidas no Brasil. Entretanto, esses autores relataram que poucas escalas foram avaliadas quanto a todas as suas qualidades psicométricas. Em adição, observa-se que as medidas de avaliação da espiritualidade e religiosidade são amplamente utilizadas na pesquisa em psiquiatria clínica e saúde mental, inclusive no contexto psicoterapêutico ${ }^{44}$, enquanto na pesquisa em Saúde Coletiva ainda há um uso discreto dessas escalas. Nesse sentido, o presente estudo buscou demonstrar que a escala P-DUREL pode ser utilizada em populações mais abrangentes, não sendo restrita a pacientes psiquiátricos ou a grupos específicos de interesse em pesquisa em psiquiatria.

O DUREL abrange as dimensões organizacional, não organizacional e intrínseca da religiosidade. Outras possíveis dimensões são o coping ou enfrentamento religioso (a forma de usar a religião para reduzir o impacto de situações estressantes) $)^{45}$, a religiosidade subjetiva (o quanto a própria pessoa se considera religiosa ${ }^{46}$ ou o quanto considera a religião importante em sua vida ${ }^{47}$ ), crenças e valores (por exemplo, crença em reencarnação e vida após a morte) e a própria filiação ou denominação religiosa à qual o indivíduo declara pertencer ${ }^{47}$. Uma abrangente revisão das diversas dimensões da religiosidade e de suas potenciais implicações à saúde é apresentada por Idler et al. ${ }^{48}$. Por sua vez, os estudos em Saúde Coletiva costumam fazer uso apenas da filiação religiosa como variável explicativa ou preditora de eventos de interesse. Entretanto, indivíduos podem declarar-se membros de alguma instituição religiosa, motivados por convenções sociais, tradições familiares ou interesses pessoais, sem que essa simples afiliação provoque ações e movimentos que influenciem seu modo de pensar, entender e interagir com o ambiente e a sociedade. $\mathrm{O}$ conceito de religiosidade expresso no instrumento P-DUREL, por sua vez, traz uma quantificação do envolvimento religioso do indivíduo dentro de domínios que podem ter profundas relações com eventos de interesse em saúde.

Utilizando uma população de usuários de serviços de atenção primária à saúde, o presente estudo mostrou uma esperada associação entre as afiliações religiosas e as dimensões do P-DUREL, em que Testemunhas de Jeová apresentam maiores escores médios da escala, seguidos de evangélicos, espíritas e católicos. Como esperado, ateus e indivíduos que creem em Deus sem possuir afiliação religiosa apresentam menores escores médios das dimensões da escala. Em adição, indivíduos com idade superior a 50 anos apresentaram 
maiores escores médios de RNO e RI, mas não há uma associação evidente entre a RO e as diferentes faixas etárias. Esse efeito pode encontrar diversas compreensões, que envolvem aspectos que não são ligados a componentes sociais. Por exemplo, Duarte et al. ${ }^{49}$ descrevem que a importância atribuída a uma religião pode representar ao idoso um mecanismo de apoio ao enfrentamento de seus problemas cotidianos, contribuindo para uma sensação de amparo. Segundo Sommerhalder e Golstein ${ }^{50}$, o envelhecer promove a reflexão sobre questões existenciais que a religião procura responder. Na Tabela 1, observa-se que os escores médios para as dimensões RO e RI da religiosidade tendem a decrescer conforme os níveis de escolaridade avançam, embora o modelo de regressão tenha evidenciado tal efeito somente para a RI (Tabela 2), após ajustamentos por sexo, faixa etária, afiliação religiosa e autopercepção do estado de saúde. Explicações para a associação negativa entre escolaridade e RI podem ser bastante complexas, envolvendo a hipótese de que pessoas com maior nível educacional são mais resistentes a dogmas religiosos e tendem a possuir um pensamento mais analítico e menos intuitivo, como sustentado por Zuckerman et al. ${ }^{51}$, ou que o uso de práticas religiosas para o enfrentamento de problemas e conflitos é mais frequente em pessoas de menor escolaridade, conforme sugerido por Seidl et al. ${ }^{52}$.

A ausência de efeitos chão e teto substanciais para as dimensões RO e RNO e a presença de um efeito teto substancial para a dimensão RI é também evidenciada no estudo de Lucchetti $^{31}$. O efeito chão substancial observado para as três dimensões do P-DUREL, quando considerados os indivíduos ateus, e para as dimensões RO e RNO, quando considerados os indivíduos que acreditam em Deus sem possuir afiliação religiosa, é esperado, assim como o efeito teto observado para evangélicos e Testemunhas de Jeová, também considerando todas as dimensões. É possível interpretar esses efeitos como uma forma de concordância entre as dimensões RO, RNO e RI da religiosidade e da afiliação ou denominação religiosa, esta também entendida como uma dimensão de religiosidade.

\section{CONCLUSÃO}

Enquanto estudos anteriores de validação do P-DUREL evidenciaram que a escala é válida para uso em populações universitárias e psiquiátricas brasileiras, o presente estudo descreveu que ela também possui adequada consistência interna e validade convergente-discriminante, considerando uma amostra representativa de uma população de usuários de serviços de atenção primária à saúde (Tabela 4). Assim, os resultados aqui obtidos sugerem que o uso do P-DUREL não é restrito à pesquisa em saúde mental, e que sua utilização pode trazer novas perspectivas para o entendimento de eventos de interesse na área da Saúde Coletiva.

\section{REFERÊNCIAS}

1. Barreto ML. Editorial: a pesquisa em Saúde Coletiva no Brasil. Cad Saúde Pública. 2003;19(2):354-55.

2. Guimarães R, Lourenço R, Cosac $S$. A pesquisa em epidemiologia no Brasil. Rev Saúde Pública. 2001;35(4):321-40.

3. Piovesan A. Pesquisa social em saúde pública. Rev Saúde Pública. 1970;4:189-205.

4. Durkheim E. Formas Elementares da Vida Religiosa. Os Pensadores. São Paulo: Ed. Abril; 1983.

5. Koenig HG, McCullough M, Larson DB. Handbook of religion and health: a century of research reviewed. New York: Oxford University Press; 2001.

6. Taunay TCD, Gondim FAA, Macêdo DS, Moreira-Almeida A, Gurgel LA, Andrade LMS, et al. Validação da versão brasileira da escala de religiosidade de Duke (DUREL). Rev Psiquiatr Clín. 2012;39(4):130-5.

7. Olinto MTA, Moreira-Filho DC. Fatores de risco e preditores para o aborto induzido: estudo de base populacional. Cad. Saúde Pública. 2006;22(2):365-75.

8. Aghababaei N. The euthanasia-religion nexus: exploring religious orientation and euthanasia attitude measures in a Muslim context. Omega (Westport). 2012-2013;66(4):333-41.
9. Ward BJ, Tate PA. Attitudes among NHS doctors to requests for euthanasia. BMJ. 1994;308(6940):1332-4

10. Oliver M, Woywodt A, Ahmed A, Saif I. Organ donation, transplantation and religion. Nephrol Dial Transplant. 2011;26(2):437-44.

11. Davis C, Randhawa G. The influence of religion on organ donation and transplantation among the Black Caribbean and Black African population-a pilot study in the United Kingdom. Ethn Dis. 2006;16(1):281-5.

12. Wallace JM Jr, Brown TN, Bachman JG, LaVeist TA. The influence of race and religion on abstinence from alcohol, cigarettes and marijuana among adolescents. J Stud Alcohol. 2003;64(6):843-8.

13. Brown TL, Parks GS, Zimmerman RS, Phillips CM. The role of religion in predicting adolescent alcohol use and problem drinking. J Stud Alcohol. 2001;62(5):696-705.

14. Lucchetti G, Koenig HG, Pinsky I, Laranjeira R, Vallada H. Religious beliefs and alcohol control policies: a Brazilian nationwide study. Rev Bras Psiquiatr. 2014;36(1):4-10.

15. Verona APDA, Dias Júnior CS. Religion and fertility among adolescents in Brazil. Rev Panam Salud Publica. 2012;31(1):25-31.

16. Benjamins MR. Does religion influence patient satisfaction? Am J Health Behav. 2006;30(1):85-91 
17. Lucchetti G, Lucchetti AL, Koenig HG. Impact of spirituality/religiosity on mortality: comparison with other health interventions. Explore (NY). 2011;7(4):234-8.

18. Linard AG, Silva FAD, Silva RM. Mulheres submetidas a tratamento para câncer de colo uterino - percepção de como enfrentam a realidade. Rev Bras Cancerol. 2002;48(4):493-8.

19. Dalgalarrondo P, Soldera MA, Correa Filho HR, Silva CAM. Religião e uso de drogas por adolescentes. Rev Bras Psiquiatr. 2004;26(2):82-90.

20. Sanchez ZM, Nappo SA. A religiosidade, a espiritualidade e o consumo de drogas. Rev Psiquiatr Clín. 2007;34(Supl. 1):73-81.

21. Panzini RG, Rocha NS, Bandeira DR, Fleck MPA. Qualidade de vida e espiritualidade. Rev Psiquiatr Clín. 2007;34(Supl. 1):105-15.

22. Koenig HG, Parkerson Jr GR, Meador KG. Religion index for psychiatric research. Am J Psychiatry. 1997;154:885-6.

23. Koenig HG, Büssing A. The Duke University Religion Index (DUREL): a five-item measure for use in epidemiological studies. Religions. 2010;1:78-85.

24. Cardoso MCS, Ferreira MC. Envolvimento religioso e bem-estar subjetivo em idosos. Psicol Cienc Prof. 2009;29(2):380-93.

25. Stroppa A, Moreira-Almeida A. Religiosidade e Saúde. In: Salgado MI, Freire G. Saúde e Espiritualidade: uma nova visão da medicina. Belo Horizonte: Inede; 2008.

26. Hoge DR. A validated intrinsic religious motivation scale. J Sci Study Relig. 1972;11(4):369-76.

27. Sherman AC, Plante TG, Simonton S, Adams DC, Harbison C, Burris SK. A multidimensional measure of religious involvement for cancer patients: the Duke Religious Index. Support Care Cancer. 2000;8(2):102-9.

28. Storch EA, Roberti JW, Heidgerken AD, Storch JB, Lewin AB, Killiany EM, et al. The Duke Religion Index: a psychometric investigation. Pastoral Psychol. 2004;53(2):175-81.

29. Moreira-Almeida A, Peres MF, Aloe F, Lotufo Neto F, Koenig HG. Versão em português da Escala de Religiosidade da Duke囚DUREL. Rev Psiq Clín. 2008;35(1):31-2.

30. Lucchetti G, Granero Lucchetti AL, Peres MF, Leão FC, MoreiraAlmeida A, Koenig HG. Validation of the Duke Religion Index: DUREL (Portuguese version). J Relig Health. 2012;51(2):579-86.

31. Martinez EZ, Santos Almeida RG, Carvalho ACD. Propriedades da Escala de Religiosidade de Duke em uma amostra de pós-graduandos. Rev Psiq Clín. 2012;39(5):180.

32. Alminhana LO, Menezes Jr A, Moreira-Almeida A. Personalidade, religiosidade e qualidade de vida em indivíduos que apresentam experiências anômalas em grupos religiosos. J Bras Psiquiatr. 2013;62(4):268-74

33. Bentley J, Ahmad Z, Thoburn J. Religiosity and posttraumatic stress in a sample of East African refugees. Ment Health Relig Cult. 2014;17(2):85-95.

34. Lucchetti G, Braguetta CC, Vallada C, Vallada H. Exploring the acceptance of religious assistance among patients of a psychiatric hospital. International Journal of Social Psychiatry. 2013;59(4):311-7.

35. Moon YS, Kim DH. Association between religiosity/spirituality and quality of life or depression among living-alone elderly in a South Korean city. Asia Pac Psychiatry. 2013;5(4):293-300.
36. Roza DL, Caccia-Bava MC, Martinez EZ. Spatio-temporal patterns of tuberculosis incidence in Ribeirão Preto, State of São Paulo, southeast Brazil, and their relationship with social vulnerability: a Bayesian analysis. Rev Soc Bras Med Trop. 2012;45(5):607-15.

37. Scheaffer RL, Mendenhall III W, Ott RL, Gerow KG. Elementary Survey Sampling. $7^{\text {th }}$ edition. Boston: Cengage Learning; 2011.

38. Griffiths DA. Maximum likelihood estimation for the beta-binomial distribution and an application to the house-hold distribution of the total number of cases of a disease. Biometrics. 1973;29:637-48.

39. Martinez EZ, Almeida RGS, Braz ACG, Carvalho ACD. Association between religiousness and blood donation among Brazilian postgraduate students from health-related areas. Rev Bras Hematol Hemoter. 2014;36(3):184-90.

40. Martinez EZ, Achcar JA. Trends in epidemiology in the 21st century: time to adopt Bayesian methods. Cad Saude Publica. 2014;30(4):703-14.

41. Bland JM, Altman DG. Cronbach's alpha. BMJ. 1997;314(7080):572.

42. Pinheiro RS, Viacava F, Travassos C, Brito ADS. Gênero, morbidade, acesso e utilização de serviços de saúde no Brasil. Cienc Saúde Colet. 2002;7(4):687-707.

43. Lucchetti G, Lucchetti ALG, Vallada H. Measuring spirituality and religiosity in clinical research: a systematic review of instruments available in the Portuguese language. Sao Paulo Med J. 2013;131(2):112-22.

44. Peres JFP, Simão MJP, Nasello AG. Espiritualidade, religiosidade e psicoterapia. Rev Psiquiatr Clín. 2007;34(1):136-45.

45. Panzini RG, Bandeira DR. Coping (enfrentamento) religioso/espiritual. Rev Psiquiatr Clín. 2007;34(1):126-35.

46. Netto SM, Moreira-Almeida A. Metodologia de Pesquisa para Estudos em Espiritualidade e Saúde. In: Santos FS, organizador. Arte de cuidar: saúde, espiritualidade e educação. Bragança Paulista; Editora Comenius; 2010. p. 182-96.

47. Moreira-Almeida A, Pinsky I, Zaleski M, Laranjeira R. Religious involvement and sociodemographic factors: a Brazilian national survey. Rev Psiquiatr Clín. 2010;37(1):12-5.

48. Idler EL, Musick MA, Ellison CG, George LK, Krause N, Ory MG, et al. Measuring multiple dimensions of religion and spirituality for health research conceptual background and findings from the 1998 General Social Survey. Res Aging. 2003;25(4):327-65.

49. Duarte YAO, Lebrão ML, Tuono VL, Laurenti R. Religiosidade e envelhecimento: uma análise do perfil de idosos do município de São Paulo. Saúde Coletiva. 2008;5(24):173-7.

50. Sommerhalder C, Goldstein LL. O papel da espiritualidade e da religiosidade na vida adulta e na velhice. In: Freitas EV, Py L, Cançado FAX, Gorzoni ML, editores. Tratado de Geriatria e Gerontologia. $2^{\mathrm{a}}$ ed. Rio de Janeiro: Guanabara Koogan; 2006. p. 1307-15.

51. Zuckerman M, Silberman J, Hall JA. The relation between intelligence and religiosity: a meta-analysis and some proposed explanations. Pers Soc Psychol Rev. 2013;17(4):325-54.

52. Seidl EMF, Tróccoli BT, Zannon CMLDC. Análise fatorial de uma medida de estratégias de enfrentamento. Psic Teor Pesq. 2001;17(3):225-34.

Recebido em: 23/12/2014 Aprovado em: 05/01/2014 